\title{
Distortions of Gaussian pulses transmitted through a transparent isotropic layer
}

\author{
Distorsión de pulsos gaussianos al atravesar una capa isótropa
}

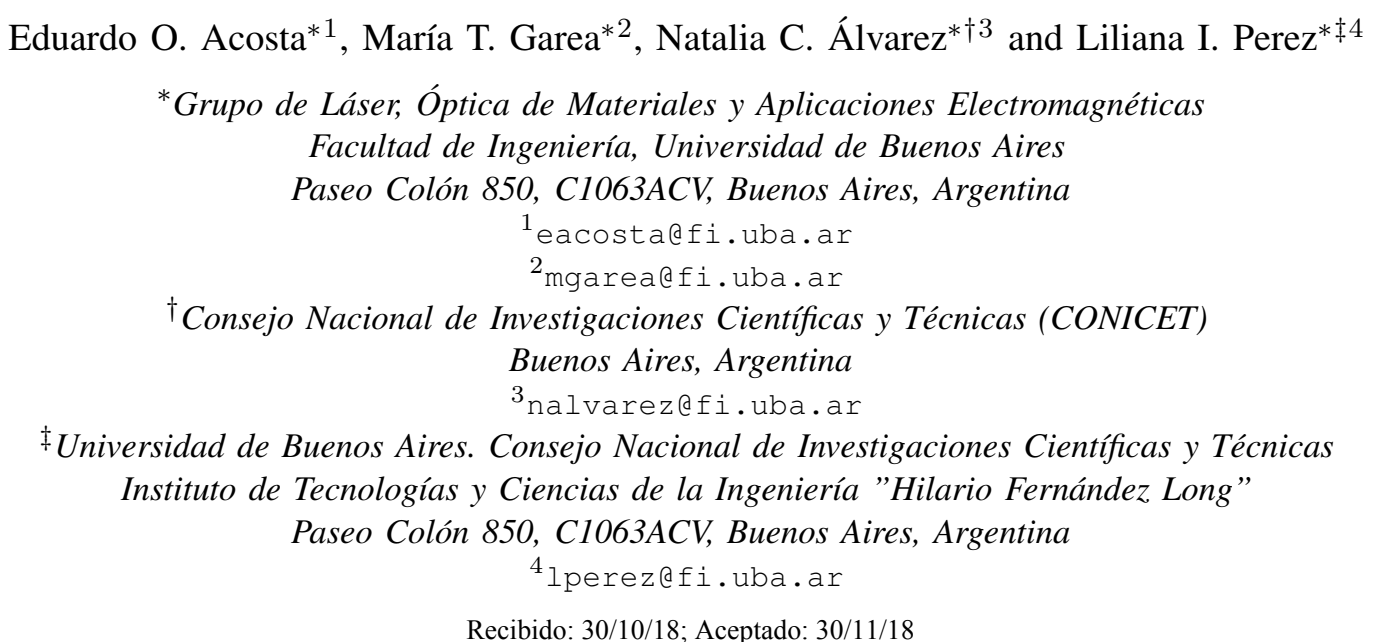

\begin{abstract}
Lasers can operate in two regimes: continuouswave mode or pulsed mode. In the simplest case, the former mode corresponds to monochromatic beams with Gaussian distribution of amplitudes (beam limited in space); whereas the latter mode corresponds to polychromatic beams with Gaussian distribution of frequencies (pulse limited in time). When the pulsed beams are reflected and refracted in different types of interfaces, they undergo peculiar distortions that bear some parallelism with those found for beams limited in space. These effects, as shown in a previous work, correspond to time delay (first order) and change of pulse width (second order). The distortions are clearly limited by the principle of causality and their interpretation, while not straightforward, emerges clearly when the associated fields are expressed in magnitude and phase. Since the analytical expressions are not simple even for the case where the pulse is transmitted through a single layer of linear, homogeneous, isotropic and transparent material, it makes it difficult to solve the inverse problem. In this work, we present an alternative analytical development that makes it possible to explicitly determine these distortion effects when a pulse impinges normally on a transparent isotropic layer immersed in a medium of analogous characteristics.
\end{abstract}

Keywords: Gaussian pulses; Geometric Optics; Phase shift

Resumen- Los láseres pueden operar en dos regímenes: modo continuo ó pulsado. En los casos más simples, el modo continuo se corresponde a haces monocromáticos con distribución de amplitudes gaussiana (es un haz limitado en el espacio); mientras que el modo pulsado corresponde a haces policromáticos con distribución gaussiana de frecuencias (pulso limitado en el tiempo). Cuando los haces pulsados se reflejan y refractan en diferentes tipos de interfaces, sufren distorsiones peculiares que tienen cierto paralelismo con los encontrados para haces limitados en el espacio. Estos efectos, como se muestra en un trabajo anterior, corresponden al retardo de tiempo (primer orden) y al cambio de ancho de pulso (segundo orden). Las distorsiones están claramente limitadas por el principio de causalidad y su interpretación, aunque no es directa, emerge claramente cuando los campos asociados se expresan en magnitud $y$ fase. Pero como las expresiones analíticas no son simples (incluso en el caso de que el pulso se transmita a través de una capa única de material lineal, homogéneo, isotrópo y transparente) se hace difícil resolver el problema inverso. En este trabajo, presentamos un desarrollo analítico alternativo que hace posible determinar explícitamente estos efectos de distorsión cuando un pulso incide normalmente en una capa isotrópica transparente sumergida en un medio de características análogas.

Palabras clave: Pulsos gaussianos; Óptica geométrica; Cambio de fase

\section{INTRODUCTION}

When a 2D finite-width monochromatic beam undergoes reflection and refraction at an planar interface between two isotropic dielectric media, it is deformed. This deformation is due to the fact that a finite beam of light is composed of a superposition of a large number of plane waves that propagate at slightly different angles of incidence with respect to the central plane wave.

Under the paraxial condition, this leads to a small correction in the Fresnel equations, producing small effects that modify the beam. The first and second order effects are lateral displacement or Goos-Hänchen effect and angular shift (first order effects) and change of focal point and change of width (second order effects). These have been studied through different approaches as energy considerations [1] [2], superposition of two or more waves with different directions of propagation [3] [4] [5] [6] [7] or moment theory [8] [9]. Each of these methods have contributed to the knowledge and determination of the different nongeometrical effects.

Nevertheless, Gaussian or quasi-Gaussian beams have been the most studied space-limited beams because they are the lowest solution of the paraxial wave equation. In order to obtain analytical expressions of these effects, usually the Tamir's generalized method (TGM) is used. This method, an extension of the Tamir's method [5], refers to the classic 
model of multiple reflections on the interfaces of an isotropic dielectric layer. The amplitudes of the successive terms of the expansion rapidly decrease, i.e., it is a rapid convergence method.

Chromatic dispersion is present in practically all optical materials and is manifested as a dependence of the phase velocity on the frequency or wavelength of light inclusive in transparent media. As known, the group velocity is the velocity that characterizes optical pulses either in dispersive or non-dispersive media as well. An optical pulse propagates at the group velocity

In the propagation, reflection and transmission of the real Gaussian pulses there can be two types of deformations: temporal and spatial. The latter corresponds to the distortion that occurs with Gaussian beams (i.e. just spatially limited) [10].

The propagation and transformation of laser pulses are fundamental problems in the fields of laser technique, optical communication, optical information processing, etc. Many works have focused on propagation through dispersive and anisotropic media [11] [12] [13]. However, there are no studies about the distortion that occurs when a pulse (only limited in time)is reflected or transmitted through nondispersive dielectric interfaces

In this work we study the distorsions that occur on a Gaussian pulse just limited in time when it is reflected or transmitted on a single layer of non-dispersive material using an analytical development alternative to TGM. The alternative method applied to these type of pulses is a generalization of the so call moment theory of light beam propagation [14] [15] [16]. Finally the results obtained with both methods are compared.

\section{INCIDENT ELECTRIC FIELD OF A GAUSSIAN PULSE}

Consider a Gaussian pulse, centered on a mean frequency $\omega_{0}$ and spectral width $\sigma$, which propagates in vacuum that impinges normally on a parallel plate of refractive index $n$ and thickness $d$ as shown in the figure 1 . The electric fields corresponding to the incident pulse $\vec{E}_{1}$ are given by

$$
\vec{E}_{1}(x, t)=\frac{\vec{E}_{0}}{\sqrt{2 \pi} \sigma} \int_{-\infty}^{+\infty} e^{-\frac{\left(\omega-\omega_{0}\right)^{2}}{2 \sigma^{2}}} e^{i \omega(x / c-t)} d \omega
$$

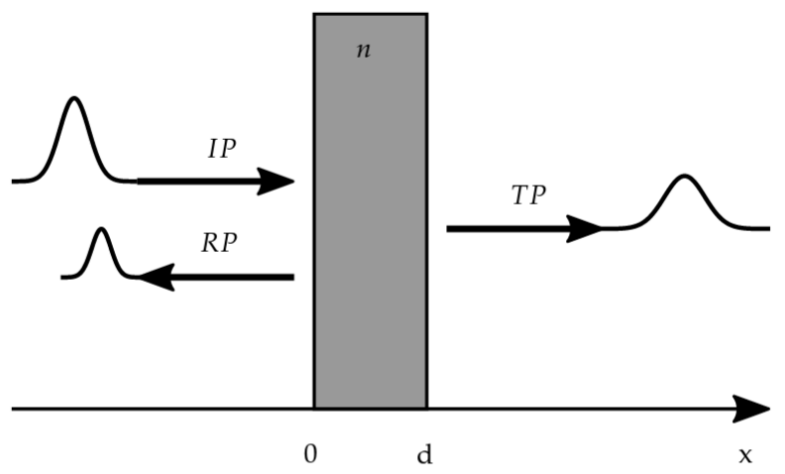

Fig. 1. Diagram of the system under analysis: incident (IP), reflected (RP) and transmitted pulses (TP)

where $\omega_{0}$ corresponds to the mean frequency. Without loss of generality we assume that the vector $\vec{E}$ has a $\hat{y}$ direction.
The integral of Eq. (1) is solved by taking $\Omega=\left(\omega-\omega_{0}\right) / \sigma$ and using the series expansion of $e^{i z}=\sum_{j=0}^{\infty}(-i)^{j} z^{j} / j$ !, replacing in Eq. (1) we obtain:

$$
\vec{E}_{1}(x, t)=\vec{E}_{0} e^{i \omega_{0}(x / c-t)} \sum_{k=0}^{\infty} \frac{(-i)^{j}}{j !} \sigma^{j}(t-x / c)^{j} Q_{j}
$$

with

$$
Q_{j}=\frac{1}{\sqrt{2 \pi}} \int_{-\infty}^{+\infty} e^{-\frac{\Omega^{2}}{2}} \Omega^{j} d \Omega
$$

The term $Q_{j}$ of the Eq. (2) is the central moment of the standard normal distribution $E\left(\Omega^{n}\right)$. By the symmetry of the normal function, the terms of odd $j$ are canceled and the moments of $j$ pairs are calculated following the equation $\mathbf{E}\left(\Omega^{2 k}\right)=(2 k) ! / 2^{k} k$. . Replacing in Eq. (2), we obtain the electric field for the incident Gaussian pulse:

$$
\vec{E}_{1}(x, t)=\vec{E}_{0} e^{i \omega_{0}(x / c-t)} e^{-\frac{\sigma^{2}}{2}(x / c-t)^{2}}
$$

\section{REFLECTED AND TRANSMITTED ELECTRIC FIELD OF A GAUSSIAN PULSE}

Assuming that the incident pulse impinges the surface normally, the electric fields corresponding to the reflected $\vec{E}_{2}$ and transmitted $\vec{E}_{3}$ pulses are given by

$$
\vec{E}_{2}(x, t)=\frac{\vec{E}_{0}}{\sqrt{2 \pi} \sigma} \int_{-\infty}^{+\infty} R(\omega) e^{-\frac{\left(\omega-\omega_{0}\right)^{2}}{2 \sigma^{2}}} e^{-i \omega(x / c+t)} d \omega
$$

$$
\vec{E}_{3}(x, t)=\frac{\vec{E}_{0}}{\sqrt{2 \pi} \sigma} \int_{-\infty}^{+\inf } T(\omega) e^{-\frac{\left(\omega-\omega_{0}\right)^{2}}{2 \sigma^{2}}} e^{i \omega(x / c-t)} d \omega
$$

where $R(\omega)$ and $T(\omega)$ are the reflection and transmission coefficients. An analytical expression of the fields can be obtained by approximating these coefficients by a polynomial series around the average frequency $\omega_{0}$ :

$$
S(\omega)=S\left(\omega_{0}\right)+\left.\sum_{k=0}^{\infty} \frac{1}{k !} \frac{\partial^{k} S}{\partial \omega^{k}}\right|_{\omega_{0}} \Delta \omega^{k}
$$

where $S$ is $R$ or $T$ and $\Delta \omega=\omega-\omega_{0}$. Replacing Eq. (6) in Eq. (4) and using the same calculation procedure as for the incident field, we obtain the reflected electric field

$$
\vec{E}_{2}(x, t)=\vec{E}_{0} e^{-i \omega_{0}(x / c+t)}\left(R\left(\omega_{0}\right) e^{-\frac{\sigma^{2}}{2}(x / c+t)^{2}}+A_{2}\right)
$$

with

$A_{2}=\left.\sum_{k=1}^{\infty} \frac{i^{k}}{k !} \frac{\partial^{k} R}{\partial \omega^{k}}\right|_{\omega_{0}} \sum_{j=0}^{\infty} \frac{(-i)^{j}(x / c+t)^{j}}{j !} \sigma^{k+j+1} \mathbf{E}\left(\Omega^{j+k}\right)$

using the expression of the moments we can obtain a simpler expression for $A_{2}$

$$
A_{2}=\left.\sum_{k=1}^{\infty} \frac{i^{k}}{k !} \frac{\partial^{k} R}{\partial \omega^{k}}\right|_{\omega_{0}} \frac{\partial^{k}}{\partial t^{k}} e^{-\frac{\sigma^{2}}{2}(x / c+t)^{2}}
$$


In the same way, replacing Eq. (6) in Eq. (5) and using the same procedure the transmitted field is written as

$\vec{E}_{3}(x, t)=\vec{E}_{0} e^{i \omega_{0}((x-d) / c-t)}\left(T\left(\omega_{0}\right) e^{-\frac{\sigma^{2}}{2}((x-d) / c+t)^{2}}+A_{3}\right)$

with

$$
A_{3}=\left.\sum_{k=1}^{\infty} \frac{i^{k}}{k !} \frac{\partial^{k} R}{\partial \omega^{k}}\right|_{\omega_{0}} \frac{\partial^{k}}{\partial t^{k}} e^{-\frac{\sigma^{2}}{2}((x-d) / c-t)^{2}}
$$

\section{COMPARISON WITH THE RESUlTS OBTAINED BY} MEANS OF TAMIR'S GENERALIZED METHOD

In the Tamir's generalized Method (TGM) the logarithm of the reflection and transmission coefficients can be replaced by their second order approximations around the mean frequency [17]

$$
\ln S(\omega)=\ln S\left(\omega_{0}\right)+\left.\frac{\partial \ln S}{\partial \omega}\right|_{\omega_{0}} \Delta \omega+\left.\frac{1}{2} \frac{\partial^{2} \ln S}{\partial \omega^{2}}\right|_{\omega_{0}} \Delta \omega^{2}
$$

Introducing Eq. (9) in Eq. (4) and integrating we obtain

$$
\vec{E}_{2}^{T G M}(x, t)=\vec{E}_{0} \frac{R(\omega)}{\sigma / \sigma_{2}} e^{-i \omega_{0}(x / c+t)} e^{-\frac{\sigma_{2}^{2}}{2}\left(-x / c-t+\tau_{2}\right)^{2}}
$$

where we define

$$
\tau_{2}=-\left.i \frac{\partial \ln R}{\partial \omega}\right|_{\omega_{0}} \text { and } \sigma_{2}^{2}=\frac{\sigma^{2}}{1-\left.\sigma^{2} \frac{\partial^{2} \ln R}{\partial \omega^{2}}\right|_{\omega_{0}}}
$$

Eq. (10) show that the pulse suffers a first order effect, corresponding to a complex time delay $\tau_{2}$ and a second order effect, corresponding to a complex change of half-width $\sigma_{2}$.

To compare both methods we truncate the series from Eq. (7) to second order $(k=2)$

$$
\vec{E}_{2}(x, t)=\vec{E}_{0} e^{-i \omega_{0}(x / c+t)} e^{-\frac{\sigma^{2}}{2}(x / c+t)^{2}} P_{2}\left(u_{2}\right)
$$

with $u_{2}=\sigma(x / c+t)$ and

$$
P_{2}(u)=R\left(\omega_{0}\right)-\left.i \sigma \frac{\partial R}{\partial \omega}\right|_{\omega_{0}} u-\left.\frac{1}{2} \sigma^{2} \frac{\partial^{2} R}{\partial \omega^{2}}\right|_{\omega_{0}}\left(u^{2}-1\right)
$$

When comparing expressions Eq. (7) and Eq. (10) it is not possible to determine if both expressions are similar.

Figure 2 shows incident pulse and reflected pulse calculated using equations (7) and (10) for $\omega_{0}=1.77 \times 10^{15} \mathrm{~s}^{-1}$; $\sigma=\omega_{0} / 500$ assuming a plate of thickness $d=22 \pi c / \omega_{0}$ and refractive index $n=1.33$; calculated for $x=0$ as a function of the dimensionless time $\omega_{0} t$. The curves calculated using both approximations show a similar shift, for the case of the TGM approximation the shift of $\omega_{0} \tau \approx 91$, while in the presented development it is $\omega_{0} \tau \approx 98$. However, the halfwidth of the half-height pulse $(\Delta)$ in both cases coincides with $\omega_{0} \Delta=607$ being 1.03 times the width of the initial pulse.

The electric field of the transmitted pulse calculated using the TGM approximation is calculated in a similar way by replacing equation Eq. (9) in Eq. (5)

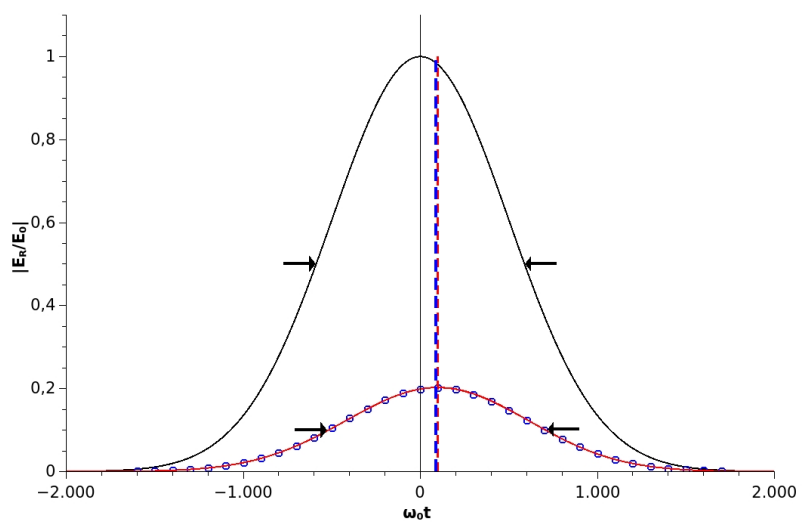

Fig. 2. Incident pulse (black), reflected pulse using the method of moments (red) and TGM (o) calculated at $x=0$

$$
\vec{E}_{3}^{T G M}(x, t)=\vec{E}_{0} \frac{S(\omega)}{\sigma / \sigma_{3}} e^{-i \omega_{0}((x-d) / c-t)} e^{-B(t)}
$$

with

$$
B(t)=\frac{\sigma_{3}^{2}}{2}\left(-(x-d) / c+t+\tau_{3}\right)^{2}
$$

where we define

$$
\tau_{3}=-\left.i \frac{\partial \ln T}{\partial \omega}\right|_{\omega_{0}} \text { and } \sigma_{3}^{2}=\frac{\sigma^{2}}{1-\left.\sigma^{2} \frac{\partial^{2} \ln T}{\partial \omega^{2}}\right|_{\omega_{0}}}
$$

In order to compare both methods we developed the Eq. (8) to second order

$$
\begin{aligned}
& \vec{E}_{3}(x, t)=\vec{E}_{0} e^{-i \omega_{0}((x-d) / c-t)} e^{-\frac{\sigma^{2}}{2}((x-d) / c-t)^{2}} P_{3}\left(u_{3}\right) \\
& \text { with } u_{3}=\sigma((x-d) / c-t) \text { and }
\end{aligned}
$$

$$
P_{3}(u)=T\left(\omega_{0}\right)-\left.i \sigma \frac{\partial T}{\partial \omega}\right|_{\omega_{0}} u-\left.\frac{1}{2} \sigma^{2} \frac{\partial^{2} T}{\partial \omega^{2}}\right|_{\omega_{0}}\left(u^{2}-1\right)
$$

In Fig. 3 shows the incident pulse and the transmitted pulse calculated using equations (15) and (13) calculated for $x=d$. In this case the curves calculated using both approximations show a similar shift $\omega_{0} \tau \approx 93$, however the pulse half-width in both cases coincides $\omega_{0} \Delta=588$ being the same as that of the initial pulse.

\section{CONCLUSion}

We show that Gaussian pulses, when reflected or transmitted on a single layer, exhibit peculiar distortions. We determine expressions for the first and second order effects: time delay and pulse width. The method used (method of moments) is an alternative to the generalized Tamir's method and extended to pulses limited in time. Although the distortions are very low at optical frequencies and begin to be noticeable at frequencies of microwaves or less we can see that the results obtained do not differ numerically from each other. Nevertheless, from the method of moments it is not simple to extract explicit expressions for such 


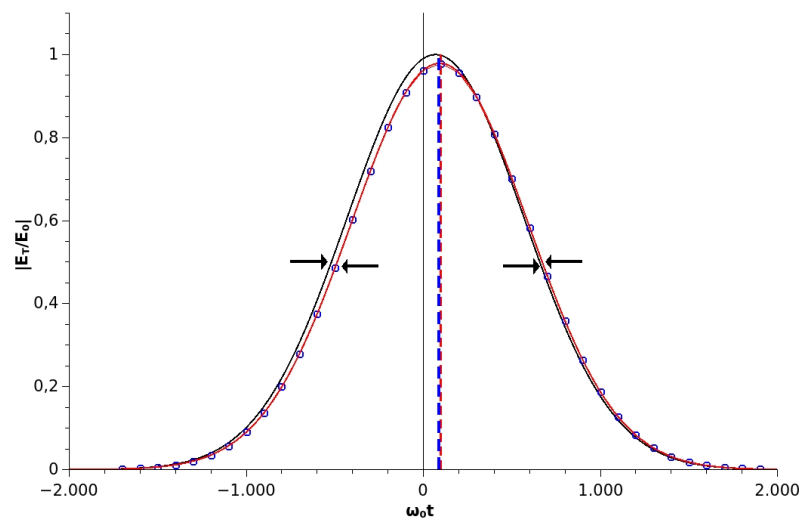

Fig. 3. Incident pulse (black), transmittes pulse using the method of moments (red) and TGM (o) calculated at $x=0$

deformations; but instead the TGM provides explicit expressions, simple to calculate. In all cases the major analytical difficulty is to calculate explicit expressions of the real and imaginary parts of the fields (or of their module and phases) when dealing with complex interfaces (several interfaces). Although Tamir's approximation is likely to be better than the second order and can easily been extended up to fourth order, the latter is easily extended to higher orders since a recurrence equation has been obtained

\section{ACKNOWLEDGMENT}

This work was supported by the Universidad de Buenos Aires under Grants UBACyT (2014-2017) 20020130100346BA, (2017- 2020) 20020160100042BA and (2017-2020) 20020160100052BA. Also, a postdoctoral grant from CONICET for one of the authors is gratefully acknowledged.

\section{REFERENCES}

[1] J. Picht, "About the vibration process that corresponds to any (astigmatic) beam," Annalen der Physik, vol. 382, no. 16, pp. 785-882, 1925. [Online]. Available: https://onlinelibrary.wiley.com/doi/abs/10.1002/andp.19253821602

[2] K. Yasumoto and Y. Oishi, "A new evaluation of the goos-hanchen shift and associated time delay," Journal of Applied Physics, vol. 54, no. 5, pp. 2170-2176, 1983. [Online]. Available: https://doi.org/10.1063/1.332395

[3] F. Noether, "About the distribution of the energy flow in total reflection," Annalen der Physik, vol. 403, no. 2, pp. 141-146, 1931. [Online]. Available: https://onlinelibrary.wiley.com/doi/abs/10.1002/andp.19314030203

[4] K. Artmann, "Calculation of the lateral displacement of the totally reflected beam," Annalen der Physik, vol. 437, no. 1-2, pp. 87-102, 1948. [Online]. Available: https://onlinelibrary.wiley.com/doi/abs/10.1002/andp.19484370108

[5] T. Tamir, "Nonspecular phenomena in beam fields reflected by multilayered media," J. Opt. Soc. Am. A, vol. 3 , no. 4, pp. 558-565, Apr 1986. [Online]. Available: http://josaa.osa.org/abstract.cfm?URI=josaa-3-4-558

[6] W. Nasalski, "Three-dimensional beam reflection at dielectric interfaces," Optics Communications, vol. 197, no. 4, pp. $217 \quad-233$, 2001. [Online]. Available: http://www.sciencedirect.com/science/article/pii/S0030401801014201

[7] L. I. Perez, R. M. Echarri, M. T. Garea, and G. D. Santiago, "Determination of nongeometric effects: equivalence between artmann's and tamir's generalized methods," J. Opt. Soc. Am. $A$, vol. 28, no. 3, pp. 356-362, Mar 2011. [Online]. Available: http://josaa.osa.org/abstract.cfm?URI=josaa-28-3-356
[8] M. A. Porras, "Moment-method evaluation of the angular and lateral shifts of reflected light beams," Optics Communications, vol. 131, no. 1, pp. 13 - 20, 1996. [Online]. Available: http://www.sciencedirect.com/science/article/pii/0030401896003379

[9] J. Alda and J. M. Rico-Garcia, "Angular shifts of paraxial beams by refraction in a plane dielectric/dielectric interface," Optics Communications, vol. 213, no. 4, pp. 229 - 239, 2002. [Online]. Available: http://www.sciencedirect.com/science/article/pii/S0030401802021168

[10] J. Diels, W. Rudolph, P. Liao, and P. Kelley, Ultrashort Laser Pulse Phenomena, ser. Optics and photonics. Elsevier Science, 2006. [Online]. Available: https://books.google.com.ar/books?id=rDQe81K0d3kC

[11] S. A. Planas, N. L. P. Mansur, C. H. B. Cruz, and H. L. Fragnito, "Spectral narrowing in the propagation of chirped pulses in singlemode fibers," Opt. Lett., vol. 18, no. 9, pp. 699-701, May 1993. [Online]. Available: http://ol.osa.org/abstract.cfm?URI=ol-18-9-699

[12] M. Rosete-Aguilar, F. Estrada-Silva, N. Bruce, C. Román-Moreno, and R. Ortega-Martínez, "Calculation of temporal spreading of ultrashort pulses propagating through optical glasses," Revista mexicana de física, vol. 54, pp. 141 - 148, 042008.

[13] P. Krehlik, "Characterization of semiconductor laser frequency chirp based on signal distortion in dispersive optical fiber," OptoElectronics Review, vol. 14, no. 2, pp. 119-124, Jun 2006. [Online]. Available: https://doi.org/10.2478/s11772-006-0015-z

[14] "Special issue on laser beam quality." Opt. Quantum Electron, vol. 24, no. 9, Sep. 1992.

[15] M. A. Porras, J. Alda, and E. Bernabeu, "Complex beam parameter and abcd law for non-gaussian and nonspherical light beams," Appl. Opt., vol. 31, no. 30, pp. 6389-6402, Oct 1992. [Online]. Available: http://ao.osa.org/abstract.cfm?URI=ao-31-30-6389

[16] P. A. Bélanger, "Beam propagation and the abcd ray matrices," Opt. Lett., vol. 16, no. 4, pp. 196-198, Feb 1991. [Online]. Available: http://ol.osa.org/abstract.cfm?URI=ol-16-4-196

[17] E. O. Acosta, N. C. Álvarez, M. T. Garea, L. Perez, and P. Sorichetti, "Deformaciones de segundo orden en la transmisión de haces y pulsos gaussianos a través de una capa isótropa," Revista elektron, vol. 2, no. $1,2018$. 